\title{
A expansão dos serviços de reabilitação no SUS à luz do arcabouço normativo federal
}

\author{
The expansion of rehabilitation services in the SUS in the light of the \\ federal normative framework
}

\section{Luciana de Assis Caetano', Rosana Ferreira Sampaio ${ }^{2}$, Luciana Assis Costa ${ }^{3}$}

http://dx.doi.org/10.11606/issn.2238-6149.v29i3p195-203

Caetano LA, Sampaio RF, Costa LA. A expansão dos serviços de reabilitação no SUS à luz do arcabouço normativo federal. Rev Ter Ocup Univ Sao Paulo. 2018 set.-dez.;29(3):195-203.

RESUMO: O estudo teve como objetivo analisar as legislações nacionais que se relacionam a criação, organização, financiamento e direcionamento dos serviços de reabilitação no país após a constituição do SUS. Trata-se de um estudo de análise documental realizado em duas etapas: busca manual dos documentos, e análise crítica destes. Foram levantadas e analisadas 56 legislações relacionadas aos serviços de reabilitação dentre leis, decretos e portarias da Casa Civil e do Ministério da Saúde. A análise das legislações se deu apoiada em quatro dimensões: concepção de deficiência que orienta os serviços de reabilitação; concepção de assistência; diretrizes para implantação dos serviços de reabilitação e procedimentos ofertados e por fim, os repasses financeiros. Os resultados apontam que a expansão dos serviços de reabilitação se deu de forma incremental, e lenta, e só a partir de 2012, de caráter estrutural, no sentido do estabelecimento de uma legislação que prevê a criação da rede de reabilitação voltada às pessoas com deficiência. Pode-se afirmar que hoje os serviços de reabilitação estão ancorados legalmente por um aparato legislativo que prevê a organização dos equipamentos, bem como os repasses para seu financiamento.

Descritores: Sistema Único de Saúde; Reabilitação/legislação \& jurisprudência; Política de saúde.
Caetano LA, Sampaio RF, Costa LA. The expansion of rehabilitation services in the SUS in the light of the federal normative framework. Rev Ter Ocup Univ Sao Paulo. 2018 SeptDec;29(3):195-203.

\begin{abstract}
The study aimed to analyze the national laws that relate to the creation, organization, financing and direction of rehabilitation services in the country after the constitution of the SUS. It is a documentary analysis study carried out in two stages: manual search of the documents, and critical analysis of these. A total of 56 legislations related to rehabilitation services among laws, decrees and ordinances of the Civil House and the Ministry of Health were collected and analyzed. Legislations analysis is divided into four dimensions: disability concept that guides the services of rehabilitation; design of assistance; guidelines for the implementation of rehabilitation services and procedures offered and, finally, financial transfer. The results indicate that the expansion of rehabilitation services occurred incrementally, and slowly, and only more recently, from 2012 onwards, of a structural nature, in the sense of establishing legislation that provides for the creation of a rehabilitation network focused on disabled people. It can be affirmed that today the rehabilitation services are anchored legally by a legislative apparatus that foresees the organization of the equipment, as well as the transfer for its financing.
\end{abstract}

Keywords: Unified Health System; Rehabilitation/legislation \& jurisprudence; Health policy.

Este estudo é parte integrante da Dissertação de Mestrado "Implementação dos Serviços de Reabilitação do SUS no Município de Belo Horizonte: avanços institucionais e desafios assistenciais".

1. Fisioterapeuta, Mestre em Ciência da Reabilitação, Universidade Federal de Minas Gerais (UFMG)- Belo Horizonte (MG), Brasil. ORCID: 0000-0002-7880-4100. E-mail: lucianaacaetano@gmail.com.

2. Doutora em Saúde Pública. Programa de Pós-graduação em Ciências da Reabilitação, Universidade Federal de Minas Gerais (UFMG) - Belo Horizonte (MG), Brasil. ORCID: 0000-0002-4775-9650. E-mail: sampaioufmg@gmail.com.

3. Doutora em Saúde Pública. Programa de Pós-graduação em Ciências da Reabilitação, Universidade Federal de Minas Gerais (UFMG) - Belo Horizonte (MG), Brasil. ORCID: 0000-0003-3664-4839. E-mail: lucianaassis.ufmg@gmail.com.

Endereço para correspondência: Rua Apucarana, 208 Apto 302B. Bairro Ouro Preto. Belo Horizonte, MG. E-mail: lucianaacaetano@ gmail.com. 


\section{INTRODUÇÃO}

S egundo a Organização Mundial de Saúde (OMS), reabilitação é "um conjunto de medidas que ajudam pessoas com deficiências ou prestes a adquirir deficiências a terem e manterem uma funcionalidade ideal na interação com seu ambiente" (p.100)1. Em linhas gerais, o termo reabilitação pode ser compreendido sob duas perspectivas: como ações voltadas ao indivíduo, tal como preconizado pela OMS, e ainda, como equipamentos que prestam este tipo de assistência ${ }^{2}$. Neste estudo, trataremos do termo reabilitação como a oferta de serviços especialmente no âmbito da Política de Saúde.

Até o final dos anos 1980, os serviços de reabilitação no país se organizavam para atender basicamente dois públicos: os trabalhadores formais, por meio dos Centros de Reabilitação Profissional vinculados ao Instituto Nacional de Assistência Médica da Previdência Social (INAMPS) ${ }^{3}$, e os serviços filantrópicos que atendiam o público com deficiência ${ }^{4}$.

Este cenário foi paulatinamente alterado, com a criação do Sistema Único de Saúde (SUS) em 1988. A partir de então, os serviços de reabilitação passaram a ser contemplados nas Políticas de Saúde, o que marca um avanço na oferta destes serviços no que se refere à universalidade de acesso. No entanto, é importante destacar que a reabilitação não obteve status de política com legislações, orçamentos e aparatos organizacionais próprios. A reabilitação passou a ser definida e organizada a partir de normatizações oriundas de diversas áreas técnicas do Ministério da Saúde (MS), que dispõem de políticas específicas como a da Pessoa com Deficiência, do Idoso, de Trauma e Violência ${ }^{5}$, e também do Trabalhador.

Assim, apesar da reabilitação ter sido encampada pela Política de Saúde, ela permanece diluída em normativas de diferentes subáreas. Verificou-se que, à exceção da política de saúde da pessoa com deficiência, que concentra o maior número de legislações associadas aos serviços de reabilitação, os demais serviços vinculados às subáreas da saúde são de caráter pontual, seletivo e não organizam uma rede de atendimento própria, o que justificou o foco deste estudo centrar na regulamentação federal da política de saúde da pessoa com deficiência.

Em relação à escolha das legislações nacionais, ressalta-se que o MS é responsável por instituir e coordenar a política nacional de saúde definindo as bases comuns a todo o território nacional e muitas vezes induzindo os outros entes da federação a implementarem serviços que integrarão a rede de saúde ${ }^{6}$. No caso da reabilitação, não é diferente: o MS tem feito uso da edição de portarias que definem e organizam os serviços de reabilitação atuando como mecanismo indutor ao condicionar a sua implementação ao repasse de recursos financeiros a estados e municípios.

"O recurso às portarias ministeriais tem sido um valioso, senão o principal instrumento de coordenação das ações nacionais de saúde, estabelecendo um arranjo no qual o Governo Federal ocupa posição central, detendo o poder de agenda e exercendo maior influência sobre processos decisórios" (p.31)7 da saúde.

A perenidade da ação pública, neste caso, a oferta de serviços de reabilitação, depende diretamente dos aspectos institucionais que os regem, como a existência de aparato legal, definição de repasses orçamentários e definição estrutural. Sendo assim, esse estudo tem como objetivo analisar as legislações nacionais, referentes à pessoa com deficiência, que se relacionam à criação, organização, financiamento e direcionamento dos serviços de reabilitação no país após a constituição do SUS. Como objetivos específicos analisar os conceitos de deficiência e reabilitação que orientam a normatização dos serviços, descrever como se encontra a regulamentação da estrutura organizacional desses serviços de reabilitação e identificar a existência de recursos destinados aos serviços de reabilitação previstos na legislação.

\section{METODOLOGIA}

Trata-se de um estudo de análise documental realizado em duas etapas: busca manual dos documentos, e análise crítica dos mesmos. A busca foi feita on-line em sites oficiais do governo brasileiro como o do MS, assembléias e portais legislativos, Diário Oficial da União, sites da pessoa com deficiência, site do Sistema de Auditoria do SUS, manuais do MS, e regulamentações revogadas citadas em legislações mais atuais sobre a pessoa com deficiência para, assim, acessar o histórico de todas as legislações relacionadas à reabilitação a partir da constituição do SUS cobrindo o período de 1989 a julho de 2017.

Foram encontradas e analisadas 56 legislações relacionadas aos serviços de reabilitação dentre leis, decretos e portarias do MS.

Após o levantamento das legislações, aquelas relacionadas às áreas da pessoa com deficiência foram colocadas em ordem cronológica e analisadas a partir de quatro dimensões: concepção de deficiência que orienta os serviços de reabilitação; concepção de assistência; diretrizes para implantação dos serviços de reabilitação e procedimentos ofertados, e repasses financeiros. 


\section{RESULTADOS E DISCUSSÃO}

\section{Concepções de Deficiência e Organização da Assistência - histórico e construções que orientam os serviços de reabilitação no SUS}

As legislações que fazem referência aos serviços de reabilitação, após a implementação do SUS, estão pulverizadas em várias políticas transversais, contudo, grande parte está associada à saúde da pessoa com deficiência. Estas foram promulgadas no período de 1989 a 2015, para ordenar e organizar as ações e os serviços ofertados a essa população específica.

Com a promulgação da Constituição de 1988, a temática da pessoa com deficiência se consolidou como pauta de governo apoiada em várias legislações da Casa Civil (Lei ${ }^{\circ} 7853 / 1989$ e Lei ${ }^{\circ} 13146 / 2015$ - Estatuto da Pessoa com Deficiência, Decreto no 3298/1999 Política Nacional para a Integração da Pessoa Portadora de Deficiência e Decreto $n^{\circ}$ 7612/2011 - Plano Viver Sem Limites) que orientam as portarias do MS responsáveis pela regulamentação das leis, estabelecendo regras e organização do funcionamento dos serviços de reabilitação na saúde ${ }^{8}$.

Por seu turno, os serviços de reabilitação, desde as primeiras portarias criadas no início da década de 1990, seguem os conceitos e diretrizes do SUS, como universalidade, integralidade, hierarquização e regionalização da assistência. É possível observar que, apesar de manter as bases do SUS originárias na Constituição, ao longo dos anos, as legislações sofrem a influência de novas concepções e conceitos veiculados em todo mundo.

A concepção de deficiência prevista nas legislações nacionais que tratam dos serviços de reabilitação foi mudando ao longo do tempo, seguindo as tendências internacionais. As primeiras legislações ( Lei $^{\circ}{ }^{0} 7853 / 1989$, Portarias MS/SNAS n ${ }^{\circ}$ 225/1992, no 303/1992 e $n^{\circ}$ 304/1992, Portaria MS/SAS n ${ }^{\circ} 116 / 1993$ e Decreto ${ }^{\circ}$ 3298/1999), traziam o conceito apresentado pela OMS a partir das "Famílias de Classificações Internacionais". Em 1976, a Classificação Internacional de Deficiências, Incapacidades e Desvantagens - CIDID- definia deficiência como uma falta ou anormalidade, incapacidade como restrição ou impossibilidade de realização de atividade de forma "normal" e desvantagem (handicap) como uma situação prejudicial que limitava ou impedia o desempenho "normal" do indivíduo 9,10.

Essa concepção de deficiência que coloca no indivíduo a causa da limitação ou impedimento e não considera a influência organizativa do ambiente para o entendimento da incapacidade e da desvantagem ${ }^{11}$, direcionou os serviços e procedimentos de reabilitação no âmbito nacional até o início dos anos 2000, com prioridade para os níveis de alta e média complexidade.

Em 1997, a OMS publicou a segunda versão da CIDID com novos conceitos: Classificação Internacional das Deficiências, Atividades e Participação: um manual da dimensão das incapacidades e da saúde-CIDDM-2. Nesta classificação foram enfatizados os contextos ambientais e as potencialidades do indivíduo, retirando o foco das incapacidades e limitações ${ }^{9}$. Com a mudança de concepção em âmbito internacional, as legislações nacionais tentaram seguir o mesmo entendimento (Portaria MS/GM n ${ }^{\circ}$ 1060/2002 e demais Portarias MS/GM até 2004).

É importante ressaltar que as legislações do final dos anos 1990 e início dos anos 2000, apresentam características de uma fase de transição tanto conceitual, como de orientação de serviços. O público beneficiário dessas legislações foi ampliado com o entendimento de que todas as pessoas que apresentassem deficiência, independente da natureza, agente causal ou grau de severidade (Decreto no 3298/1999) assim como aqueles com doenças crônicas que resultassem em desvantagem ou incapacidade (Portaria MS/GM n ${ }^{\circ}$ 1060/2002) seriam beneficiários dos processos de reabilitação? .

A criação da Rede Estadual de Assistência a Pessoa Portadora de Deficiência Física (Portaria MS/ $\mathrm{GM} \mathrm{n}^{\circ}$ 818/2001), continuou estruturando os serviços de reabilitação na média e alta complexidade. Essa portaria priorizou os serviços de reabilitação dirigidos às pessoas com deficiência física e estabeleceu os serviços de menor complexidade às unidades ambulatoriais.

No ano seguinte, a portaria que cria a Política Nacional de Saúde da Pessoa Portadora de Deficiência (Portaria MS/GM n ${ }^{\circ}$ 1060/2002) avançou em relação aos princípios da reabilitação e forma de assistência. Além da ampliação do público, ela reforçou a criação de redes de serviços de saúde nos diversos níveis de complexidade, priorizando a assistência integral a pessoa com deficiência e trazendo a atenção básica e a Reabilitação Baseada na Comunidade (RBC) para o cenário da reabilitação?.

A RBC foi proposta pela OMS com o objetivo de valorizar e desenvolver o potencial da pessoa com deficiência e da comunidade em que ela vive como agente no processo de reabilitação e inclusão socia ${ }^{12}$. Porém, a operacionalização da $\mathrm{RBC}$, só seria possível a partir da introdução de uma equipe de reabilitação na atenção básica, o que foi feito com a criação do Núcleo de Apoio a Saúde da Família (NASF) em 2008.

A legislação que cria o NASF (Portaria MS/GM $n^{\circ} 154 / 2008$ ) trouxe a Classificação Internacional de Funcionalidade, Incapacidade e Saúde (CIF), apresentada pela OMS em 2001, como evolução da CIDDM-2. "A CIF substitui o enfoque negativo da deficiência e da incapacidade 
por uma perspectiva positiva, considerando as atividades que um indivíduo que apresenta alterações de função e/ ou da estrutura do corpo pode desempenhar, assim como sua participação social" $\left(\right.$ p.187) ${ }^{10}$. A funcionalidade e incapacidade humana passaram a ser entendidas como condições determinadas pelo contexto ambiental e não por aspectos físicos e orgânicos. A mudança da concepção de deficiência internacionalmente induziu a mudança da concepção de reabilitação em âmbito nacional, levando a uma nova forma de organização e oferta de serviços para as pessoas com deficiência.

A criação do NASF foi um marco por introduzir os conceitos da CIF no cenário nacional e por possibilitar a introdução dos serviços de reabilitação na atenção básica. A legislação do NASF trouxe novamente a RBC, reforçando a integralidade dos serviços de saúde para a pessoa com deficiência, em especial a reabilitação. A partir da legislação do NASF, influenciada pela Convenção Internacional sobre os Direitos das Pessoas com Deficiência de 2007, houve a modificação da nomenclatura de "pessoa portadora de deficiência", como era utilizado até então nas legislações, para "pessoa com deficiência", termo atual. A mudança na terminologia reforça a mudança conceitual, pois a pessoa com deficiência não porta ou carrega a deficiência como sugeria o termo anterior, ela tem deficiência, sendo assim o termo "pessoa com deficiência" mais adequado ${ }^{13}$.

A Convenção foi assinada em 2007 pelo Brasil, e introduzida no ordenamento jurídico nacional com valor de emenda constitucional ${ }^{14}$. Ela reforça a mudança do modelo médico para o modelo social no qual o foco da limitação é o ambiente e não a deficiência em si, como preconizado pela $\mathrm{CIF}^{15}$.

As legislações relacionadas à saúde da pessoa com deficiência que seguiram no período de 2008 até 2015, última legislação analisada, mantiveram a concepção de deficiência e, consequentemente, de reabilitação trazida pela CIF. No cenário nacional, o Conselho Nacional de Saúde do Ministério da Saúde, definiu em 2012, por meio da Resolução MS/CNS n ${ }^{\circ}$ 452/2012 pela utilização da CIF nas legislações do SUS.

Pode-se, todavia, afirmar que, as legislações que formulam e regulamentam os serviços de reabilitação no SUS acompanharam, no âmbito normativo, as mudanças conceituais e filosóficas, ancoradas no modelo social, que orientam a compreensão e uso do termo deficiência tal como seguem as orientações internacionais.

\section{Os Serviços de reabilitação e os repasses financeiros previstos na normativa do SUS}

O financiamento das Políticas Públicas é condição crucial para garantia da concretude da ação planejada pelo Estado. Além disso, a existência de financiamento para determinada política demonstra quais serão as prioridades do governo ${ }^{16}$. Daí percebe-se a importância de uma norma legal atrelada ao repasse financeiro para a sua execução.

A Figura 1 apresenta a síntese das legislações que regulamentam a implementação dos serviços e procedimentos de reabilitação no SUS previstas nas normativas de saúde para as pessoas com deficiência, no período de 1989 a 2015.

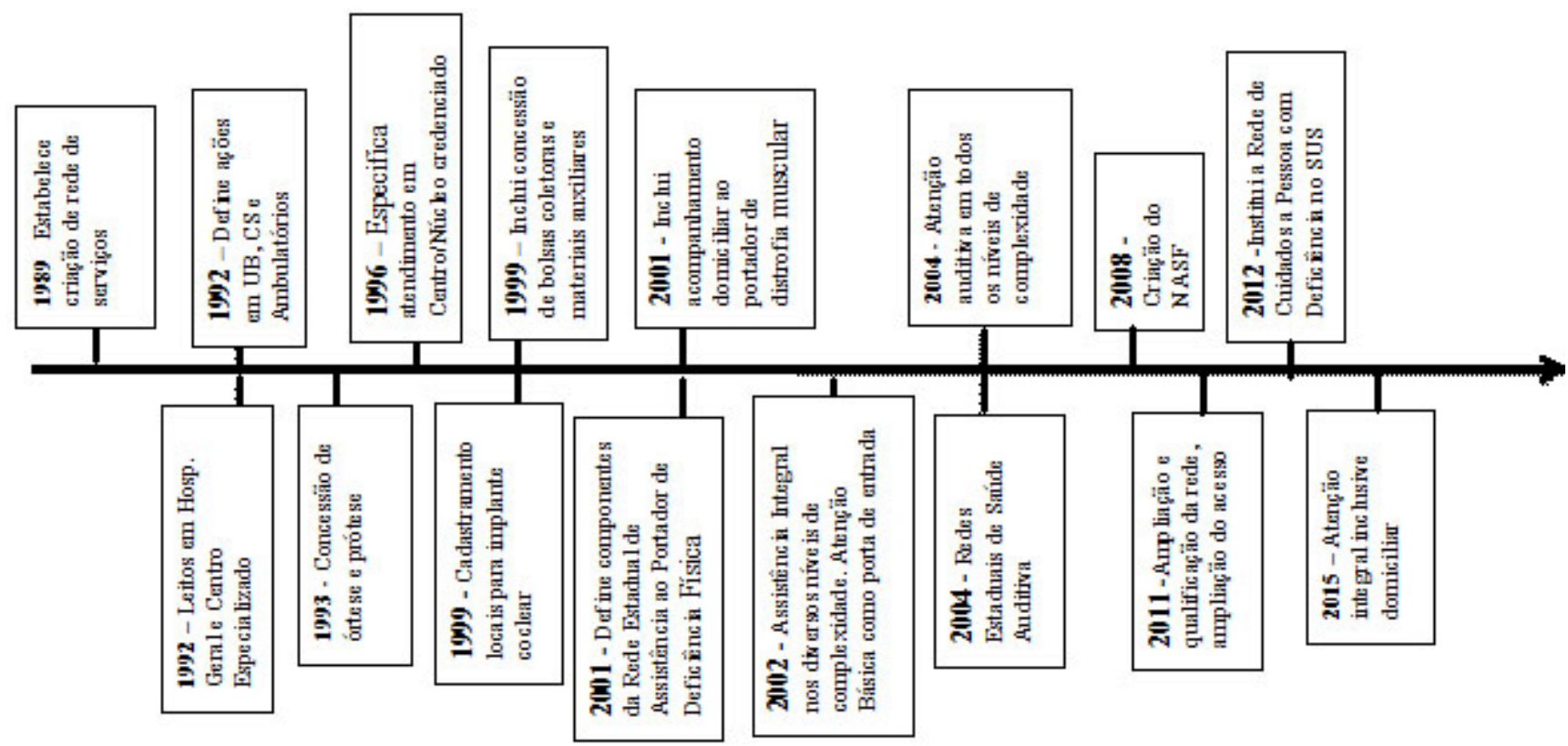

Figura 1: Linha do Tempo com as Legislações que apresentam as diretrizes para implementação dos serviços e procedimentos de reabilitação no SUS 
No final da década de 1980, tal como apresentado na Figura 1, a primeira legislação que tratou dos serviços de saúde direcionados às pessoas com deficiência, foi a lei criada pela Casa Civil da Presidência da República que previa o estabelecimento de uma rede de serviços especializados em reabilitação e habilitação, além de ações de promoção e prevenção da deficiência (Lei n $\left.{ }^{\circ} 7853 / 1989\right)$. Contudo, como é próprio das leis, a normativa não trouxe detalhamentos de quais seriam os serviços e as fontes de financiamento dos mesmos, sendo assim, objeto de portarias do MS na década de $1990^{8}$.

Desde o início da década de 1990, as portarias que orientaram os serviços de reabilitação voltados à pessoa com deficiência trataram prioritariamente da inclusão desses serviços na média e alta complexidade, ambulatoriais e hospitalares. Estas portarias previam a inclusão dos serviços nas Tabelas dos Sistemas de Informação Ambulatorial (SIA/ SUS) e Sistemas de Informação Hospitalar (SIH/SUS) para o repasse financeiro. Dentre os serviços incluídos nas tabelas de financiamento do SUS, estavam os leitos hospitalares para reabilitação em hospitais gerais e em hospitais especializados, centros especializados e ambulatórios de reabilitação, e ainda concessão de próteses, órteses e meios auxiliares de locomoção (Portarias MS/SNAS n ${ }^{\circ} 225 / 1992$, e $\mathrm{n}^{\text {os }} 303$ a $306 / 1992$, Portarias MS/SAS n ${ }^{\circ} 116 / 1993$, ${ }^{\circ}$ 146/1993, nº 211/1996, no 584/1999 e Portaria MS/GM n 1278/1999).

Neste período, foi mantida a lógica de financiamento do sistema de saúde anterior ao SUS que privilegiava o pagamento por produção e os serviços hospitalares e ambulatoriais ${ }^{17}$. Essa forma de pagamento por produção para os serviços de reabilitação seguia o cenário mais amplo de saúde no Brasil instituído pela Norma Operacional Básica de $1991(\mathrm{NOB} / 91)^{18}$.

A forma de financiamento dos serviços começou a ser modificado a partir da edição da NOB/93. Os municípios passaram a receber recursos do Fundo Nacional de Saúde para o Fundo Municipal de Saúde, e por isso ter maior autonomia no pagamento dos prestadores de serviços ambulatoriais e hospitalar, públicos e privados ${ }^{18}$.

Com a edição da NOB/96, novos mecanismos de transferências entre os entes federados foram apresentados. Além disso, a NOB/96 aumentou a participação percentual de transferências fundo a fundo de recursos federais para estados e municípios reduzindo a remuneração por procedimentos e valorizando a atenção básica. No entanto, os serviços de reabilitação continuaram a receber o repasse por procedimentos por ainda estarem atrelados ao componente especializado da saúde ofertado em níveis ambulatorial e hospitalar ${ }^{18}$.
Em 2001, com o intuito de equalizar alocação de recursos para a saúde em geral, foi editada a Norma Operacional da Assistência a Saúde (NOAS/2001) que estabeleceu a regionalização como expediente de hierarquização dos serviços de saúde e de alcance de maior equidade. A partir da NOAS/2001 desenha-se a implementação das redes de assistência em saúde já mencionada desde a primeira legislação para a pessoa com deficiência em 1989 (Lei no $^{0}$ 7853/1989) ${ }^{19}$.

Para possibilitar a implementação da Rede de Assistência a Pessoa com Deficiência nos estados e municípios, determinada pela Portaria $\mathrm{MS} / \mathrm{GM} \mathrm{n}^{\circ} 818 / 2001$, o MS alterou, a partir da Portaria MS/SAS n ${ }^{\circ} 185 / 2001$, a descrição dos procedimentos constantes na tabela SIASUS incluindo-os nos procedimentos ambulatoriais de alta complexidade/custo (APAC-SIA). Aumenta, neste caso, a possibilidade de oferta dos serviços ambulatoriais e hospitalares para construção da rede estadual de reabilitação $0^{20}$.

A ideia de rede de serviços, assim como a valorização da atenção básica permaneceu nas legislações que se seguiram. Em 2002 a Portaria MS/GM n ${ }^{\circ}$ 1060/2002 reiterou o papel da atenção básica como a porta de entrada para os serviços de saúde da pessoa com deficiência, juntamente com os serviços de emergência de pronto atendimento. $\mathrm{Na}$ atenção secundária os Serviços de Reabilitação ambulatoriais assumiram a denominação de Centros de Referência em Reabilitação (CRR) para o atendimento especializado a pessoa com deficiência ${ }^{9}$.

No entanto, pode-se afirmar que os serviços de reabilitação previstos nas legislações da década de 1990 até os anos 2008 se expandiram de forma incremental. Isto pode ser visto na inclusão pontual de procedimentos e serviços especializados, dirigidos a públicos específicos, tais como pessoas com distrofia muscular progressiva, deficiência mental, deficiência física, autismo, e deficiência auditiva, sendo todos os serviços vinculados ao repasse financeiro por procedimentos (Portarias MS/GM n ${ }^{\circ} 1531 / 2001, n^{\circ}$ 1635/2002, no 2073/2004).

No cenário nacional da saúde, no período 2003-2006 manteve-se o incentivo à atenção básica. Contudo, ainda não existia uma rede de serviços que ultrapassasse os limites municipais para possibilitar o atendimento nos demais níveis da assistência. Assim, nesse contexto, foi instituído o Pacto pela Saúde, em 2006, que introduziu fortemente a estratégia de regionalização por meio dos Colegiados de Gestão Regional, atualmente Comissões Intergestores Regionais $(\mathrm{CIR})^{21}$. Além disso, foram definidas três dimensões no Pacto pela Saúde: Pacto pela Vida, Pacto em defesa do SUS e Pacto de Gestão. O Pacto pela Vida definiu como 
prioridade a consolidação e a qualificação da Estratégia Saúde da Família, instituída pelo governo federal desde 1994, como modelo para atenção básica e centro ordenador das redes de atenção à saúde no SUS ${ }^{22}$. O Pacto pela Saúde modificou a forma de repasse dos recursos financeiros para blocos de financiamento.

No caso dos serviços de reabilitação, esta lógica do financiamento por blocos irá refletir, de forma mais contundente, após a criação do NASF que insere a reabilitação na atenção básica com seu financiamento compondo o bloco da atenção básica, e mais tardiamente, com a criação dos Centros Especializados em Reabilitação (CER), compondo o bloco de média e alta complexidade ambulatorial e hospitalar.

Para proporcionar a atenção integral, em 2008, a rede de assistência a pessoa com deficiência foi reforçada com a inserção da reabilitação na atenção básica a partir do NASF. Assim, a Rede de Assistência à Pessoa com Deficiência passou a ofertar serviços de reabilitação nos três níveis de complexidade. O NASF trouxe o serviço de reabilitação para fora dos ambulatórios e hospitais, orientado pelo conceito ampliado de deficiência apresentado pela CIF. Além disso, o repasse financeiro para as equipes de NASF é feito por valores mensais fixos, de acordo com o tipo de NASF credenciado (Portaria MS/GM no 548/2013). Esse repasse se dava fundo a fundo, para o do bloco de financiamento da atenção básica, mantendo a característica de financiamento trazida pelo Pacto de 2006.

Nos níveis secundário e terciário, o repasse financeiro ainda se manteve por procedimentos, vinculados aos blocos de financiamento de média e alta complexidade ambulatorial e hospitalar, até a Portaria MS/GM n ${ }^{\circ}$ 793/2012 criar o Centro Especializado em Reabilitação (CER) que passa a ser financiado por custeio e capital. O CER é um dos equipamentos de reabilitação criado para compor a rede de reabilitação a partir do Plano Viver sem Limites de 2011 (Decreto $n^{\circ}$ 7612/2011). Com o Plano a temática da pessoa com deficiência é novamente colocada em evidência como pauta de governo. Vale destacar que as legislações instituídas pela Casa Civil possuem grande peso político, e isso acompanhado de dotação orçamentaria para a implementação dos serviços atuou como mecanismo de pressão e indução a organização de uma rede de serviços para a pessoa com deficiência no SUS.

Assim, um ano depois, em 2012, o MS lançou a Portaria MS/GM n ${ }^{\circ} 793 / 2012$ que instituiu um marco na organização estrutural dos serviços de reabilitação. Dito de outra forma, os serviços deixaram de ser ofertados de forma incremental e desarticulada, como aconteceu ao longo dos anos anteriores, e passaram a ser ofertados em uma rede com os pontos e serviços definidos e articulados nos três níveis de atenção. A portaria definiu a Rede de Cuidados para a Pessoa com Deficiência com os seguintes componentes: na atenção básica o NASF e atenção odontológica, na atenção secundária os estabelecimentos habilitados em apenas um Serviço de Reabilitação, Centro Especializado em Reabilitação (CER), a Oficina Ortopédica e o Centro de Especialidades Odontológicas (CEO), e por último, na atenção terciária a atenção hospitalar e de urgência e emergência, sendo prevista a garantia da integralidade do cuidado a partir da articulação entre os equipamentos e o acesso a cada ponto de atenção de forma regulada.

A proposta do CER é atender as pessoas com deficiência auditiva, física, intelectual, visual, ostomia e múltiplas deficiências em uma mesma estrutura, podendo, inclusive, se constituir como referência regional, se for pactuado no plano de ação da CIR. A forma de financiamento por incentivos financeiros de investimento e custeio (Portaria MS/GM no 835/2012) representou um avanço importante para os serviços de reabilitação. Isto implica que o recurso está atualmente vinculado ao atendimento mais amplo oferecido ao usuário e não aos procedimentos realizados, reforçando a atenção integral prevista na concepção ampliada de assistência do SUS ${ }^{23}$.

É possível inferir que somente a partir de 2012, mais especificamente após a Portaria MS/GM n ${ }^{\circ} 835 / 2012$, o MS passou a financiar a ampliação da oferta de serviços de reabilitação por meio de incentivos financeiros de investimentos e de custeio para o componente da Atenção Especializada da Rede de Cuidados à Pessoa com Deficiência, seja para implantação de novos serviços e qualificação de serviços de reabilitação já existentes ${ }^{23}$.

Contudo é importante lembrar que a expansão dos serviços de reabilitação no SUS foi paulatina e lenta, e só mais recentemente, de caráter estrutural, no sentido da regulamentação da rede de reabilitação voltada as pessoas com deficiência. Os CRR de 2002 coexistem com os CER de 2012, inclusive mantendo duas lógicas distintas de financiamento dos serviços de reabilitação, por procedimento e por custeio, respectivamente. Essa duplicidade da forma de remuneração dos serviços reflete um momento de mudanças, no qual a concepção de deficiência e reabilitação foram ampliadas, contudo, os mecanismos remuneratórios ainda estão em transição.

Em 2015, a última legislação que trata dos serviços de reabilitação para a pessoa com deficiência, instituída em forma de lei pela Casa Civil, reafirma a importância do tema no ordenamento jurídico nacional, mas não institui novo serviço ou financiamento para a área. Essa lei reforça a atenção integral a saúde com oferta de serviços em todos 
os níveis de complexidade, e reitera a importância das ações de prevenção da deficiência, da universalidade do acesso, da oferta de serviços articulados entre si e organizados em Redes de Atenção à Saúde. As Redes de Atenção à Saúde são o atual modelo organizativo de atenção à saúde do SUS, sendo consolidadas na Portaria de Consolidação MS/GM $n^{0} 3 / 2017$.

Assim, mesmo diante de todos os impasses estruturais enfrentados pelo SUS, é possível afirmar que as legislações analisadas, demonstraram a importância da Política da Pessoa com Deficiência na organização, expansão, definição e financiamento dos serviços de reabilitação ofertados pelo SUS.

Contudo é importante salientar, no contexto mais geral da política de saúde, no que se refere ao financiamento e a transferência dos recursos federais para os governos subnacionais, que em 2017, a Portaria MS/GM n ${ }^{\circ}$ 3992/2017 extinguiu o repasse por blocos de financiamento (atenção básica, média e alta complexidade ambulatorial e hospitalar, assistência farmacêutica, vigilância em saúde e gestão do SUS) e o substituiu por apenas dois blocos: bloco de custeio, que detém a quase totalidade dos recursos federais, e bloco de investimento ${ }^{24}$.

Várias críticas foram emitidas sobre a mudança no repasse da saúde, sendo as principais relativas ao enfraquecimento do MS como ordenador de políticas estruturantes do SUS e a limitação da sua função a simples repassador de recursos aos estados e municípios. Destaca-se ainda a possível exacerbação do conflito de interesses no âmbito local, com risco de perda de investimento em ações estruturantes do sistema, como atenção básica e vigilância a saúde ${ }^{25}$.

No geral, esta mudança cria um ambiente de incertezas e barganhas políticas que fragiliza o sistema, no sentido de não garantia de vinculação do investimento às reais necessidades de saúde e fortalecimento do SUS. Os serviços de reabilitação podem ser diretamente impactados por este processo de repasse financeiro, pois os colocam na disputa por recursos com as demais ações e procedimentos da saúde, em todos os níveis e especialidades, aumentando assim, o risco de retrocesso na expansão da oferta de serviços.

\section{CONSIDERAÇÕES FINAIS}

A introdução e manutenção da temática do deficiente na agenda de governo brasileiro, conquistada pelo empenho dos movimentos sociais, das organizações não governamentais, das militâncias das famílias e das pessoas com deficiência, possibilitou a instituição legal de uma Rede de Serviços de Reabilitação dentro das Redes de Atenção à Saúde do SUS, de tamanha importância e complexidade.

Além do acompanhamento das alterações relativas ao SUS, as modificações apresentadas nas legislações da pessoa com deficiência ao longo dos anos são multifatoriais e incluem também as mudanças de concepção de saúdedoença, deficiência e assistência nos contextos nacional e internacional.

Vale ressaltar que os serviços de reabilitação no SUS, por meio dos decretos e portarias federais, estão ancorados legalmente por um aparato legislativo que prevê a organização dos equipamentos, bem como os repasses para seu financiamento. Este aparato institucional é um indicativo de avanço e estruturação dos equipamentos de reabilitação na saúde, e atua como mecanismo indutor para que estados e municípios, no contexto de descentralização e regionalização da política de saúde, se responsabilizem pela implementação da rede de reabilitação.

Todavia, com a recente mudança na forma de repasse dos recursos para a saúde no contexto de restrição financeira e orçamentária, constata-se a redução do papel indutor do MS e consequente aumento dos espaços de conflito e barganha no nível local, no que tange a destinação dos recursos da saúde. Sendo assim, cabe aos representantes dos movimentos sociais, dos gestores, coordenadores e técnicos dos serviços de reabilitação maior articulação em defesa do SUS, e especificamente, da área de reabilitação, quiçá, no sentido de unificar as várias políticas que regulam os serviços de reabilitação na saúde para o fortalecimento da área.

Participação dos autores: Luciana de Assis Caetano: concepção da pesquisa, busca de dados, análise e interpretação dos dados, e redação. Luciana Assis Costa: concepção e delineamento da pesquisa, análise e interpretação dos dados, redação e revisão. Rosana Ferreira Sampaio: revisão. Todos os autores participaram da aprovação da versão final do artigo para publicação 


\section{REFERÊNCIAS}

1. Organização Mundial da Saúde (OMS). Relatório mundial sobre a deficiência/World Report on Disability. São Paulo: Secretaria dos Direitos da Pessoa com Deficiência; 2012.

2. Brasil. Ministério da Saúde. Saúde da pessoa com deficiência: diretrizes, políticas e ações. Reabilitação [citado 02 jul. 2018]. Disponível em: http://portalms.saude.gov.br/saude-para-voce/ saude-da-pessoa-com-deficiencia\#reabilitacao.

3. Paulus Júnior A, Cordoni Júnior L. Políticas públicas de saúde no Brasil. Rev Espaço Saúde 2006;8(1):13-9.

4. Ribeiro CTM, Ribeiro MG, Araújo AP, Mello LR, Rubim LC, Ferreira JES. O sistema público de saúde e as ações de reabilitação no Brasil. Rev Panam Salud Publica. 2010;28(1):43-8. Disponível em: https://scielosp.org/pdf/ rpsp/2010.v28n1/43-48/pt.

5. Cardoso LGRA. Estudo sobre a distribuição dos serviços de reabilitação: o caso do estado do Rio de Janeiro [dissertação]. Brasília: Fundação Oswaldo Cruz - FIOCRUZ; 2004.

6. Santos L. Região de saúde e suas redes de atenção: modelo organizativo-sistêmico do SUS. Cienc Saude Coletiva. 2017;22(4):1281-9. doi.org/10.1590/141381232017224.26392016 .

7. Menicucci TMG, Costa LA, Machado JA. Pacto pela saúde: aproximações e colisões na arena federativa. Cienc Saude Coletiva. 2018;23(1):29-40. doi.org/10.1590/141381232018231.17902015 .

8. Brasil. Ministério da Saúde. Secretaria-Executiva. Coordenação-Geral de Inovação Gerencial. Manual de orientação: elaboração de portarias no Ministério da Saúde. Versão preliminar. Brasília: Ministério da Saúde; 2010. (Série A. Normas e Manuais Técnicos.) [citado 11 ago. 2018]. Disponível em: http://bvsms.saude.gov.br/bvs/publicacoes/ manual_de_orientacao_elaboracao_portarias.pdf.

9. Brasil. Ministério da Saúde (BR). Portaria $n^{\circ} 1060$, de 5 de junho de 2002. Aprova a Política Nacional de Saúde da Pessoa Portadora de Deficiência [citado 2 jul. 2018]. Disponível em: http://bvsms.saude.gov.br/bvs/saudelegis/gm/2002/ prt1060_05_06_2002.html.

10. Farias N, Buchalla CM. A Classificação Internacional de Funcionalidade, Incapacidade e Saúde da Organização Mundial da Saúde: conceitos, usos e perspectivas. Rev Bras Epidemiol. 2005;8(2):187-93. doi.org/10.1590/S1415790X2005000200011.

11. Mângia EF, Muramoto MT, Lancman S. Classificação Internacional de Funcionalidade e Incapacidade e Saúde (CIF): processo de elaboração e debate sobre a questão da incapacidade. Rev Ter Ocup Univ São Paulo. 2008;19(2):12130. doi.org/10.11606/issn.2238-6149.v19i2p121-130
12. Maior IL. Reabilitação baseada na comunidade. Uma proposta viável para o Brasil. Acta Fisiatr. 1996;3(2):7-8. Disponível em: http://www.revistas.usp.br/actafisiatrica/ article/view/101997.

13. Curitiba, Paraná. Prefeitura Municipal. Assessoria Especial de Assistência à Pessoa com Deficiência. Terminologia. Terminologia sobre a pessoa que tem deficiência. Curitiba; 2013 [citado 23 ago. 2018]. Disponível em: http://www. pessoacomdeficiencia.curitiba.pr.gov.br/conteudo/ terminologia/116.

14. Brasil. Decreto $n^{\circ} 6949$, de 25 de agosto de 2009. Promulga a Convenção Internacional sobre os Direitos das Pessoas com Deficiência e seu Protocolo Facultativo, assinados em Nova York, em 30 de março de 2007 [citado 12 jul. 2018]. Disponível em: http://www.planalto.gov.br/ccivil_03/ ato2007-2010/2009/decreto/d6949.htm.

15. Brasil. Secretaria Especial dos Direitos da Pessoa com Deficiência. Convenção sobre os Direitos da Pessoa com Deficiência. Brasília; 2010 [citado 23 ago. 2018]. Disponível em: http://www.pessoacomdeficiencia.gov.br/app/publicacoes/ convencao-sobre-os-direitos-das-pessoas-com-deficiencia.

16. Salvador E. Fundo público e o financiamento das políticas sociais no Brasil. Serv Soc Rev. 2012;14(2):1-22. doi. org/10.5433/1679-4842.2012v14n2p4.

17. Fundação Getúlio Vargas (FGV). Centro de Pesquisa e Documentação de História Contemporânea do Brasil (CPDOC). Instituto Nacional de Assistência Médica da Previdência Social (INAMPS). Verbetes [citado 11 jun. 2017]. Disponível em: http://www.fgv.br/cpdoc/acervo/dicionarios/ verbete-tematico/instituto-nacional-de-assistencia-medica-daprevidencia-social-inamps.

18. Scatena JHG, Tanaka OY. Os instrumentos normalizadores (NOB) no processo de descentralização da saúde. Saúde Soc. 2001;10(2):47-74. doi.org/10.1590/S010412902001000200005.

19. Brasil. Ministério da Saúde. Portaria no 95 , de 26 de janeiro de 2001. Aprova, na forma do anexo desta portaria, a Norma Operacional da Assistência à Saúde - NOAS-SUS 01/2001 que amplia as responsabilidades dos municípios na atenção básica; define o processo de reorganização da assistência; cria mecanismos para o fortalecimento da capacidade de gestão do sistema único de saúde e procede a atualização dos critérios de habilitação de estados e municípios [citado 2 jul. 2018]. Disponível em: http://bvsms.saude.gov.br/bvs/saudelegis/ gm/2001/prt0095_26_01_2001.html.

20. Brasil. Ministério da Saúde. Secretaria de Assistência à Saúde. Manual de legislação em saúde da pessoa portadora de deficiência. Brasília: Ministério da Saúde; 2003. (Série B. 
Caetano LA, et al. A expansão dos serviços de reabilitação no SUS. Rev Ter Ocup Univ Sao Paulo. 2018 set.-dez.;29(3):195-203.

Textos Básicos de Saúde). Disponível em: http://bvsms.saude. gov.br/bvs/publicacoes/manual_legis_port_def.pdf.

21. Machado RR. Políticas de saúde no Brasil: um pouco da história. Rev Saúde Pública (Santa Catarina). 2012;5(3):95104. Disponível em: http://revista.saude.sc.gov.br/index.php/ inicio/article/view/145/175.

22. Brasil. Ministério da Saúde. Secretaria de Atenção à Saúde. Departamento de Atenção Básica. Política Nacional de Atenção Básica. 4a ed. Brasília: Ministério da Saúde; 2007. v.4. (Série E. Legislação de Saúde/Série Pactos pela Saúde 2006).

23. Brasil. Ministério da Saúde. Portaria $n^{\circ} 835$, de 25 de abril de 2012. Institui incentivos financeiros de investimento e de custeio para o Componente Atenção Especializada da Rede de Cuidados à Pessoa com Deficiência no âmbito do
Sistema Único de Saúde [citado 12 jul. 2018]. Disponível em: http://bvsms.saude.gov.br/bvs/saudelegis/gm/2012/ prt0835_25_04_2012.html.

24. Brasil. Ministério da Saúde. Portaria no 3992, de 28 de dezembro de 2017. Altera a Portaria de Consolidação $n^{0}$ 6/ GM/MS, de 28 de setembro de 2017, para dispor sobre o financiamento e a transferência dos recursos federais para as ações e os serviços públicos de saúde do Sistema Único de Saúde [citado 12 jul. 2018]. Disponível em: http://bvsms. saude.gov.br/bvs/saudelegis/gm/2017/prt3992_28_12_2017. html.

25. JUSBRASIL. Financiamento da saúde [citado 25 ago. 2018]. Disponível em: https://cd.jusbrasil.com.br/noticias/585609431/ financiamento-da-saude-e-tema-de-audiencia-na-quinta. 